\title{
IGF-1R: SUMO-ing its weight in chemoresistant colorectal cancer
}

\author{
Ajay Goel ${ }^{*}, 1$ \\ ${ }^{1}$ Center for Gastrointestinal Research and Center for Translational Genomics and Oncology, Michael A Ramsay Chair in Cancer \\ Genomics, Baylor Scott and White Research Institute and Charles A. Sammons Cancer Center, 3410 Worth Street, Suite 610, \\ Dallas, TX 75246, USA
}

Post-translational modification of proteins by members of the small ubiquitin-related modifier (SUMO) protein family regulates multiple cellular processes, including transcription, replication, chromosome segregation and DNA repair, in various human diseases including cancer (Geiss-Friedlander and Melchior, 2007). The SUMO proteins include three ubiquitin-like peptidic modifiers; SUMO-1, -2 and -3 . Their conjugation to substrates occurs through heterodimeric SUMO-activating E1 enzyme (AOS1/UBA2), a SUMO-conjugating E2 enzyme UBC9 (encoded by UBE2I) and various E3 protein ligases (e.g., PIAS1, PIAS2, PIAS3, PIAS4 and RANBP2) that facilitate reversible binding of SUMO proteins to the lysine residues of the target protein (Kerscher et al, 2006).

Although sumoylation controls many cellular functions, one well-recognised and established role is in the regulation of transcription through the modification of histones, transcription factors, chromatin-modifying enzymes, and basal transcription machinery. Dysregulation of the SUMO pathway has been observed in various cancers, and is often associated with adverse patient outcomes (Driscoll et al, 2010). Emerging evidence suggests that targeting sumoylation could be a potential therapeutic approach for cancer treatment. In this context, a specific panel of transcription factors implicated in the epithelial-mesenchymal transition (EMT) and in chemotherapeutic resistance, such as p53, MDM2, NF- $\kappa \mathrm{B}$, and ZEB2 (Du et al, 2016), as well as tyrosine kinase receptors such as IGF-1R (Sehat et al, 2010), has been shown to be directly targeted by SUMO-mediated conjugation.

In this issue of the British Journal of Cancer, Codony-Servat et al (2017) report a novel observation that phosphorylated nuclear IGF$1 \mathrm{R}$ (nIGF-1R) is expressed in $\sim 20 \%$ of metastatic colorectal cancers (mCRC) and 50\% of patients harboring mutations within the BRAF gene. In these subsets of patients, the levels of phosphorylated nIGF$1 \mathrm{R}$ in pre-treated metastases were markedly increased compared with their matched untreated primary tumours. Moreover, the authors demonstrated that high expression of nIGF-1R significantly correlated with poor overall survival in CRC patients.
To make sense of these clinical findings, the authors performed functional studies and successfully garnered supporting evidence that chemoresistant CRC cell lines displayed significantly higher levels of nIGF-1R expression. The potential molecular mechanism underlying the translocation of IGF-1R into the nucleus was explored using CRC cells treated with various chemotherapeutic drugs, rendering them chemoresistant. Codony-Servat et al (2017) observed that the protein inhibitor of activated STAT3 (PIAS3) was the key mediator contributing to IGF-1R nuclear sequestration, pointing to an essential role of PIAS3, a SUMO E3 protein ligase, in this process.

Another intriguing feature of this study was the complexity of the 'BRAF-like' phenotype in CRC patients. Such a phenotype was defined by the presence of bona fide BRAF mutations in mCRC patients, as well as the presence of a gene-expression signature in a subset of patients that lacked $B R A F$ mutations, which was very similar to the patients with $B R A F$ mutations. In fact, both groups of patients with mCRC have previously demonstrated resistance to cetuximab treatment (Popovici et al, 2012). This BRAF-like phenotype often results in the upregulation of various genes implicated in sumoylation, including: RANBP2, an E3-SUMO ligase implicated in kinetochore function during mitosis (Vecchione et al, 2016); the nuclear internalisation of IGF-1R (Packham et al, 2015); and the activation of splicing genes such as the GTPase-an active form of RAC1 and RAC1b, which promotes Cyclin D1 and NF-KB activity (Matos et al, 2008). Conversely, the downregulation of sumoylation-associated genes such as AXIN-2, CDX2 and RNF43 is observed, owing to hypermethylation of their promoter regions in the presence of point mutations (Bond et al, 2016), as illustrated in Figure 1.

This study by Codony-Servat et al (2017) is provocative and raises several important questions. First, is the expression of RANBP2 and/or PIAS3 upregulated more in BRAF-mutant mCRC patients compared with KRAS-mutant or double wild-type genotypes? If so, what is the underlying rationale for such a 


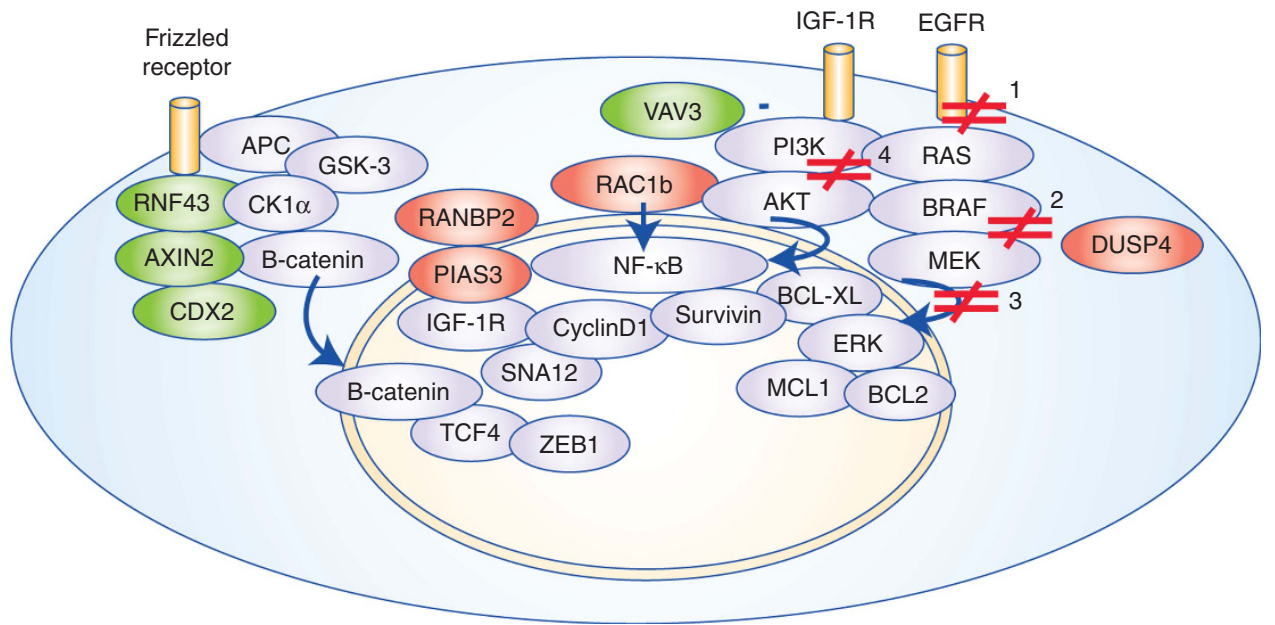

Figure 1. Routes to targeted therapy resistance in BRAF-like phenotype patients. In addition to the presence of bona fide BRAF mutations, a substantial percentage of colorectal cancer patients with KRAS mutations, as well as those with double wild-type genotypes (2 WT) are enriched with a 'BRAF-like' phenotype. Such a phenotype potentially prevents sensitivity to: (1) EGFR inhibitors (panitumumab and cetuximab); (2) BRAF inhibitors (vemurafenib, dabrafenib and encorafenib); (3) MEK inhibitors (trametinib, cobimetinib, binimetinib and selumetinib); and (4) PI3K inhibitors (alpelisib and buparlisib). As illustrated in this figure, the BRAF-like phenotype overcomes AKT/MEK inhibition by directly targeting the NF-kB transcription factor or Cyclin D1 by overexpression of SUMO proteins or RAC1b. Upregulated genes are shown in red circles, while green circles depict downregulated genes in CRC patients with a BRAF-like phenotype.

BRAF-like phenotype, which certainly seems to be involved in chemoresistance in such patients? Second, the increase in expression of PIAS3 and nIGF-1R was higher in chemotherapy pre-treated cell lines than naive cell lines when later treated with ganitumab or dasatinib-is this important? Finally, is the phosphorylated nIGF-1R just a (surrogate) biomarker for the BRAF-like phenotype or does it actually play an active role in chemotherapy and targeted therapy-mediated resistance? Although the last question would require a careful evaluation in future studies, it is already known that nIGF-1R functions as a transcription co-factor for LEF-1, which activates the expression of cyclin D1 and AXIN-2; as well as for histone H3 through recruitment of Brg1 and SNAI2 expression (Warsito et al, 2016). Of note, in this study using HeLa cells, phosphorylated nIGF-1R which was induced upon IGF-1 stimulation, was inhibited with IGF-1R kinase inhibition (Warsito et al, 2016). The finding that IGF-1R expression increases in the nucleus following ganitumab treatment in pre-treated colorectal cancer cells is quite striking and could, at least partially, help provide an explanation for its therapeutic failure in pre-treated colorectal cancer patients (Van Cutsem et al, 2014). These findings have important clinical implications as these reinforce the importance for understanding the complexity of second-line therapy for treating CRC patients (e.g., mechanism of action of ganitumab).

In conclusion, the study by Codony-Servat et al (2017) sets the stage for important treatment decision making. Recently, vinorelbine demonstrated pre-clinical activity in RANBP2 addicted BRAF-like CRC cell lines (Vecchione et al, 2016). In addition, SUMOylation inhibitors (Bogachek et al, 2016; Wagner et al, 2015) and curcumin have the potential to reverse EMT- and NF-kBmediated chemotherapeutic resistance, and nuclear internalisation of IGF-1R, respectively. Therefore, a rational step would be to explore the combinatorial efficacy of these agents in pre-treated mCRC patients with phosphorylated nIGF-1R overexpression. Other strategies worth considering might include the combination of these drugs with BRAF and MEK inhibitors, in pre-treated $B R A F$-mutant patients. The ultimate golden nugget to glean from a study such as this would be that in the era of precision medicine, the identification of robust biomarkers that could help delineate specific phenotypes will be crucial for optimal drug development in mCRC. In other words, we should have realistic aspirations of solving one piece of the puzzle at a time, rather than hoping for the big prize anytime soon.

\section{ACKNOWLEDGEMENTS}

The present work was supported by the CA72851, CA181572, CA184792, CA187956 and CA202797 grants from the National Cancer Institute, National Institute of Health; RP140784 from the Cancer Prevention Research Institute of Texas; grants from the Sammons Cancer Center and Baylor Foundation, as well as funds from the Baylor Scott and White Research Institute, Dallas, TX, USA.

\section{CONFLICT OF INTEREST}

The author declares no conflict of interest.

\section{REFERENCES}

Bogachek MV, Park JM, De Andrade JP, Lorenzen AW, Kulak MV, White JR, Gu VW, Wu VT, Weigel RJ (2016) Inhibiting the SUMO pathway represses the cancer stem cell population in breast and colorectal carcinomas. Stem Cell Rep 7(6): 1140-1151.

Bond CE, McKeone DM, Kalimutho M, Bettington ML, Pearson SA, Dumenil TD, Wockner LF, Burge M, Leggett BA, Whitehall VL (2016) RNF43 and ZNRF3 are commonly altered in serrated pathway colorectal tumorigenesis. Oncotarget 7(43): 70589-70600.

Codony-Servat JCM, Asensio E, Montironi C, Martínez-Cardús A, Marín-Aguilera M, Horndler C, Martínez-Balibrea E, Rubini M, Jares P, Reig O, Victoria I, Gaba L, Martín-Richard M, Alonso V, Escudero P, Fernández-Martos C, Feliu J, Méndez JC, Méndez M, Gallego J, Salud A, Rojo F, Castells A, Prat A, Rosell R, García-Albéniz X, Camps J, Maurel J (2017) Nuclear IGF-1R predicts chemotherapy and targeted therapy 
resistance in metastatic colorectal cancer. Br J Cancer 117(12): 1777-1786.

Driscoll JJ, Pelluru D, Lefkimmiatis K, Fulciniti M, Prabhala RH, Greipp PR, Barlogie B, Tai YT, Anderson KC, Shaughnessy Jr JD, Annunziata CM, Munshi NC (2010) The sumoylation pathway is dysregulated in multiple myeloma and is associated with adverse patient outcome. Blood 115(14): 2827-2834.

Du L, Li YJ, Fakih M, Wiatrek RL, Duldulao M, Chen Z, Chu P, Garcia-Aguilar J, Chen Y (2016) Role of SUMO activating enzyme in cancer stem cell maintenance and self-renewal. Nat Commun 7: 12326.

Geiss-Friedlander R, Melchior F (2007) Concepts in sumoylation: a decade on. Nat Rev Mol Cell Biol 8(12): 947-956.

Kerscher O, Felberbaum R, Hochstrasser M (2006) Modification of proteins by ubiquitin and ubiquitin-like proteins. Annu Rev Cell Dev Biol 22: 159-180.

Matos P, Oliveira C, Velho S, Goncalves V, da Costa LT, Moyer MP, Seruca R, Jordan P (2008) B-Raf(V600E) cooperates with alternative spliced Raclb to sustain colorectal cancer cell survival. Gastroenterology 135(3): 899-906.

Packham S, Warsito D, Lin Y, Sadi S, Karlsson R, Sehat B, Larsson O (2015) Nuclear translocation of IGF-1R via p150(Glued) and an importin-beta/ RanBP2-dependent pathway in cancer cells. Oncogene 34(17): 2227-2238.

Popovici V, Budinska E, Tejpar S, Weinrich S, Estrella H, Hodgson G, Van Cutsem E, Xie T, Bosman FT, Roth AD, Delorenzi M (2012) Identification of a poor-prognosis BRAF-mutant-like population of patients with colon cancer. J Clin Oncol 30(12): 1288-1295.
Sehat B, Tofigh A, Lin Y, Trocme E, Liljedahl U, Lagergren J, Larsson O (2010) SUMOylation mediates the nuclear translocation and signaling of the IGF-1 receptor. Sci Signal 3(108): ra10.

Van Cutsem E, Eng C, Nowara E, Swieboda-Sadlej A, Tebbutt NC, Mitchell E, Davidenko I, Stephenson J, Elez E, Prenen H, Deng H, Tang R, McCaffery I, Oliner KS, Chen L, Gansert J, Loh E, Smethurst D, Tabernero J (2014) Randomized phase $\mathrm{Ib} / \mathrm{II}$ trial of rilotumumab or ganitumab with panitumumab versus panitumumab alone in patients with wild-type KRAS metastatic colorectal cancer. Clin Cancer Res 20(16): 4240-4250.

Vecchione L, Gambino V, Raaijmakers J, Schlicker A, Fumagalli A, Russo M, Villanueva A, Beerling E, Bartolini A, Mollevi DG, El-Murr N, Chiron M, Calvet L, Nicolazzi C, Combeau C, Henry C, Simon IM, Tian S, in 't Veld S, D’Ario G, Mainardi S, Beijersbergen RL, Lieftink C, Linn S, Rumpf-Kienzl C, Delorenzi M, Wessels L, Salazar R, Di Nicolantonio F, Bardelli A, van Rheenen J, Medema RH, Tejpar S, Bernards R (2016) A vulnerability of a subset of colon cancers with potential clinical utility. Cell 165(2): 317-330.

Wagner T, Kiweler N, Wolff K, Knauer SK, Brandl A, Hemmerich P, Dannenberg JH, Heinzel T, Schneider G, Kramer OH (2015) Sumoylation of HDAC2 promotes NF-kappaB-dependent gene expression. Oncotarget 6(9): 7123-7135.

Warsito D, Lin Y, Gnirck AC, Sehat B, Larsson O (2016) Nuclearly translocated insulin-like growth factor 1 receptor phosphorylates histone $\mathrm{H} 3$ at tyrosine 41 and induces SNAI2 expression via Brg1 chromatin remodeling protein. Oncotarget 7(27): 42288-42302. 